\title{
Terpenoid biosynthesis in Arabidopsis attacked by caterpillars and aphids: effects of aphid density on the attraction of a caterpillar parasitoid
}

\author{
Anneke Kroes $^{1} \cdot$ Berhane T. Weldegergis $^{1} \cdot$ Francesco Cappai $^{1} \cdot$ Marcel Dicke $^{1}(\mathbb{D} \cdot$ \\ Joop J. A. van Loon ${ }^{1}$
}

Received: 23 April 2017 / Accepted: 12 October 2017 / Published online: 20 October 2017

(c) The Author(s) 2017. This article is an open access publication

\begin{abstract}
One of the responses of plants to insect attack is the production of volatile organic compounds that mediate indirect defence of plants by attracting natural enemies of the attacking herbivores. Herbivore-induced plant volatiles (HIPVs) include terpenoids that play key roles in the attraction of natural enemies. Crosstalk between phytohormonal signalling pathways is well known to affect the regulation of plant defences, including the emission of HIPVs. Thus, simultaneous feeding on the same plant by caterpillars and aphids, can affect the attraction of parasitoids by the plant compared to single insect attack. The role of aphid density in the regulation of HIPV emission by plants under dual attack has not been studied previously. Here, we investigated the attraction of Diadegma semiclausum, a parasitoid of the Diamondback moth Plutella xylostella, to volatiles emitted by Arabidopsis thaliana plants, simultaneously attacked by host caterpillars, and by the non-host aphid Brevicoryne brassicae. Our study shows that the effect of aphid infestation on parasitoid attraction is influenced by the density of the aphids. Biosynthesis and emission of $(E, E)-\alpha$-farnesene could be linked to the observed preference of $D$. semiclausum parasitoids for the HIPV blend emitted by plants dually infested by caterpillars and aphids at a high density compared to dually infested plants with a low aphid density.
\end{abstract}

Communicated by Moshe Inbar.

Electronic supplementary material The online version of this article (doi:10.1007/s00442-017-3985-2) contains supplementary material, which is available to authorized users.

Marcel Dicke

marcel.dicke@wur.nl

1 Laboratory of Entomology, Wageningen University, P.O. Box 16, 6700 AA Wageningen, The Netherlands
Parasitoids such as D. semiclausum are important enemies of herbivorous insects and a better understanding of how plants express indirect defence mechanisms in response to multiple insect attack will provide important knowledge on plant-herbivore-parasitoid interactions under multiple stress conditions.

Keywords Diadegma semiclausum $\cdot$ Herbivore-induced plant volatiles · Indirect defence $\cdot$ Multiple attack $\cdot$ Terpene synthase

\section{Introduction}

When facing an attack by herbivorous insects, plants may respond with structural, molecular and chemical defence mechanisms (Dicke and Van Poecke 2002; Kessler and Baldwin 2002; Schoonhoven et al. 2005; Howe and Jander 2008). One of the chemical responses to insect attack is the production of an array of volatile organic compounds that mediate indirect plant defence by attracting natural enemies of the attacking herbivores (Heil 2008; Dicke and Baldwin 2010). Herbivore-induced plant volatiles (HIPVs) include terpenoids, green leaf volatiles (GLVs) and volatile methyl esters of phytohormones (e.g. methyl salicylate and methyl jasmonate) (Arimura et al. 2005; Mumm and Dicke 2010). The emission of HIPVs may vary depending on the attacking herbivore species, density or developmental stage (Clavijo McCormick et al. 2012; Cai et al. 2013; Pashalidou et al. 2015), and parasitoids and predators use specific blends of HIPVs as cues to locate their herbivore hosts or prey feeding on the plant (De Boer et al. 2004; De Rijk et al. 2013). Therefore, the composition of the HIPV blend plays an important role in the attraction of natural enemies to herbivore-infested plants. For a few herbivore-plant-natural enemy systems 
particular volatile compounds have been found to affect the attraction of predators and parasitoids to an HIPV blend. For instance, the volatile compounds $(E, E)-4,8,12$-trimethyltrideca-1,3,7,11-tetraene $((E, E)$-TMTT) and methyl salicylate (MeSA) increased the preference of the predatory mite Phytoseiulus persimilis for prey-infested lima bean plants (De Boer et al. 2004). Herbivore-induced MeSA decreased the attraction of the parasitoid Diadegma semiclausum (Snoeren et al. 2010), whereas the herbivore-inducible terpene alcohol linalool attracts this parasitoid (Houshyani et al. 2013). Other volatile compounds that could play key roles in the attraction of D. semiclausum, a parasitoid of the specialist caterpillar Plutella xylostella (Ohara et al. 2003), are the tetranor-diterpene $(E, E)$-TMTT and the sesquiterpene $(E, E)$ - $\alpha$-farnesene. These volatiles are released as part of an HIPV blend emitted by leaves of Arabidopsis thaliana in response to P. xylostella feeding (Herde et al. 2008; Huang et al. 2010).

Not only feeding by caterpillars, but also other types of herbivory, such as aphid feeding, induce the release of terpenoids (Du et al. 1998; Dicke et al. 2003). A family of terpene synthase (TPS) genes was identified in the genome of $A$. thaliana (Aubourg et al. 2002; Aharoni et al. 2003). Terpenes are grouped based on the number of carbon atoms they contain, such as monoterpenes $\left(\mathrm{C}_{10}\right)$, sesquiterpenes $\left(\mathrm{C}_{15}\right)$ and diterpenes $\left(\mathrm{C}_{20}\right)$ (Gershenzon and Croteau 1991). The monoterpene alcohol linalool was shown to be produced by terpene synthase 10 (TPS10) in A. thaliana (Ginglinger et al. 2013). Furthermore, two closely related terpene synthase genes, TPSO2 and TPSO3, are responsible for the formation of the monoterpene $(E)$ - $\beta$-ocimene in A. thaliana ecotype Wassilewskija and the sesquiterpene $(E, E)-\alpha$-farnesene in ecotype Col-0, respectively (Fäldt et al. 2003; Huang et al. 2010). The (E,E)-geranyllinalool synthase TPS04 regulates an important step in the biosynthesis of the tetranor-diterpene (E,E)-TMTT in A. thaliana (Herde et al. 2008). The extensive knowledge of genes involved in the biosynthesis of HIPV in A. thaliana, the availability of well-characterized mutants and knowledge of tritrophic interactions involving this model plant of molecular genetics, makes this plant a suitable object for the present study (van Poecke et al. 2001; van Poecke 2007; Herde et al. 2008, Huang et al. 2010; Tholl and Lee 2011).

The induction of plant volatile biosynthesis is regulated by two main plant defence signalling pathways, the jasmonic acid (JA) and salicylic acid (SA) pathways (Ozawa et al. 2000; Arimura et al. 2005; Pieterse et al. 2012). The JAsignalling pathway regulates the biosynthesis of volatile terpenoids and GLVs (Dicke and Van Poecke 2002), whereas MeSA is synthesized in plants from SA (Chen et al. 2003; Liu et al. 2010). It is well established that leaf-chewing herbivores, such as caterpillars, induce especially JA-mediated defence responses, while phloem-feeding insects, such as aphids, trigger mainly the SA- as well as the JA-signalling pathway (De Vos et al. 2005; Stam et al. 2014). When caterpillars and aphids simultaneously feed on the same plant, a common event in nature, crosstalk between both signalling pathways may affect the regulation of plant defences (Stam et al. 2014). In addition, it is known that phloem-feeding herbivores such as aphids induce lower levels of HIPV emission compared to chewing herbivores (Turlings et al. 1998; Rodriguez-Saona et al. 2003; Ali and Agrawal 2012; Truong et al. 2014). Consequently, multiple herbivores feeding on plants interact indirectly through plant-mediated effects and this may alter the emission of HIPVs (Rodriguez-Saona et al. 2003; Dicke et al. 2009; Ponzio et al. 2013) such that it affects the attraction of predators and parasitoids by the plant, compared to single insect attack (Zhang et al. 2009; Erb et al. 2010; Zhang et al. 2013). For example, herbivory by the phloem-feeding whitefly Bemisia tabaci interfered with indirect defences of $A$. thaliana to $P$. xylostella caterpillars. For this interference by B. tabaci intact JA- and ethylene signalling was needed (Zhang et al. 2013). In addition, volatiles emitted by lima bean plants simultaneously infested by the whitefly $B$. tabaci and the spider mite Tetranychus urticae were less attractive to predatory mites compared to T. urticae-infested plants. This effect on the attraction of the predatory mite was the result of a reduction in JA-mediated emission of the monoterpene (E)- $\beta$-ocimene (Zhang et al. 2009). The same study showed that plant-mediated interference by whiteflies with indirect defence against spider mites was aphid-density dependent (Zhang et al. 2009).

Herbivore density influences the intensity of feeding damage and, consequently, may modulate interactions between plants and multiple insect attacks (Kroes et al. 2015). Therefore, herbivore density may also influence the attractiveness of herbivore-infested plants to parasitoids and predators. However, knowledge about how multiple herbivory influences the composition of volatile blends and, thus, attraction of parasitoids or predators, is limited (Ponzio et al. 2013).

This study addressed the effects of dual herbivory on induced indirect plant defence, by investigating volatile blend composition and expression of volatile-biosynthesis genes of plants attacked by single or multiple herbivores. In addition, effects of differences in HIPV emission on the attraction of parasitoids were assessed by using wellcharacterized mutant plants. We investigated indirect defence responses of A. thaliana wild-type plants and volatile-biosynthesis mutants when dually infested by $P$. xylostella caterpillars and Brevicoryne brassicae aphids compared to plants infested by $P$. xylostella caterpillars alone. The plants were infested with either a low or a high aphid density to study aphid-density-dependent effects on plant-mediated interactions between $P$. xylostella and its parasitoid D. semiclausum. We assessed the responses of the parasitoid to HIPVs emitted by dually infested plants 
and by caterpillar-infested plants. To better understand the underlying mechanisms of induced indirect defence to multiple insect attack, the expression profile of genes important for the biosynthesis of plant volatiles and volatile compounds emitted were linked to the behavioural responses of the parasitoid.

\section{Materials and methods}

\section{Plants and growth conditions}

Plants of Arabidopsis thaliana ecotype Columbia-0 (Col-0) were used as wild type. Seeds of a mutant defective in the biosynthesis of methyl salicylate, bsmt1 (benzoic acid and salicylic acid carboxyl methyltransferase 1; SALK_140496c; Snoeren et al. (2010)) were obtained from the European Arabidopsis Stock Centre (NASC, Nottingham, United Kingdom). Seeds of mutants defective in the biosynthesis of linalool, tps10 [terpene synthase 10; Ginglinger et al. (2013)] and of $(E, E)$ - $\alpha$-farnesene, tps03 [terpene synthase03; Huang et al. (2010)] were kindly provided by Thierry Delatte (Laboratory of Plant Physiology, Wageningen University, The Netherlands) and Dorothea Tholl (Department of Biological Sciences, Virginia Polytechnic Institute and State University, USA). Seeds of both wild-type and mutant plants were sown in autoclaved $\left(80^{\circ} \mathrm{C}\right.$ for $4 \mathrm{~h}$ ) potting soil (Lentse potgrond, Lent, The Netherlands). After 10-14 days of growth, plants were transferred to individual pots $(5 \mathrm{~cm}$ diameter) containing soil from the same source. Plants were cultivated in a growth chamber at $21 \pm 2{ }^{\circ} \mathrm{C}$ under an $8 \mathrm{~L}: 16 \mathrm{D}$ cycle [200 $\mu \mathrm{mol} \mathrm{m}{ }^{-2} \mathrm{~s}^{-1}$ photosynthetic active radiation (PAR) light intensity] and $60 \pm 10 \%$ relative humidity (RH). Fiveto six-week-old plants were used in the experiments. During the experiments, all plants remained in the vegetative state.

\section{Insects}

Both the cabbage aphid, B. brassicae L. (Hemiptera: Aphididae), and the diamondback moth, P. xylostella L. (Lepidoptera: Yponomeutidae), were reared on Brussels sprouts plants (Brassica oleracea var. gemmifera cv Cyrus) at $22 \pm 1{ }^{\circ} \mathrm{C}, 50-70 \% \mathrm{RH}, 16 \mathrm{~L}: 8 \mathrm{D}$ cycle. The parasitoid $D$. semiclausum Hellén (Hymenoptera: Ichneumonidae) was reared on P. xylostella feeding on Brussels sprouts plants at $22 \pm 1{ }^{\circ} \mathrm{C}, 60-70 \% \mathrm{RH}, 16 \mathrm{~L}: 8 \mathrm{D}$ cycle. Newly emerged wasps were collected and kept in a cage supplemented with $6-10 \%$ sugar water solution in a climate cabinet at $21 \pm 1{ }^{\circ} \mathrm{C}$ with a 16L:8D cycle. In all experiments, female parasitoids were naïve, i.e. without oviposition experience, 3-10 days old and mated.

\section{Olfactory responses of Diadegma semiclausum}

Preference of $D$. semiclausum parasitoids was analysed in a dual-choice test performed in a Y-tube olfactometer. The Y-tube olfactometer consisted of two 5-L glass jars which were each connected to one arm of a glass Y-tube. Incoming charcoal-filtered compressed air regulated at a flow of $2 \mathrm{~L} \min ^{-1}$ was led into each of the two glass jars containing an odour source (four A. thaliana plants). Prior to placing a plant in one of the jars, the pot of the plant was carefully wrapped in aluminium foil.

At the start of the behavioural assay, a single female parasitoid was released at the base of the Y-tube. Behaviour of the parasitoid was observed in the Y-tube olfactometer for 10 min and its choice for either odour source was recorded when the parasitoid spent at least $15 \mathrm{~s}$ beyond a line marked $2 \mathrm{~cm}$ from the end of each Y-tube arm. Parasitoids that did not choose within the observation period were excluded from the statistical analysis. After five parasitoids were tested, the position of the odour sources was exchanged to exclude positional bias in the setup. In total four sets of plants and 45-60 parasitoids were tested per combination of odour source. Each set of plants was tested on a different day with a new set of parasitoids. Each parasitoid was only tested once.

As odour source, four $A$. thaliana plants were subjected to one of the following treatments:

1. Uninfested control (undamaged).

2. Infested with two second-instar (L2) P. xylostella caterpillars (indicated as ' $P$. xylostella' infestations).

3. Simultaneously infested with five adult $B$. brassicae aphids, 'low density' (LD), and two P. xylostella L2 caterpillars (indicated as 'dual' infestations).

4. Simultaneously infested with 25 adult $B$. brassicae aphids, 'high density' (HD), and two P. xylostella L2 caterpillars (indicated as 'dual' infestations).

Insects were allowed to feed freely on the plants. Individual plants were placed in a plastic container (diameter $8 \mathrm{~cm} \times$ height $14 \mathrm{~cm}$ ), covered with gauze cloth and closed with elastic bands. Containers were randomly distributed in a tray (12-15 containers per tray). Trays were placed in a growth chamber with a $16 \mathrm{~L}: 8 \mathrm{D}$ cycle $\left(200 \mu \mathrm{mol} \mathrm{m}^{-2} \mathrm{~s}^{-1}\right.$ PAR), at $21 \pm 2{ }^{\circ} \mathrm{C}$ and $50-70 \%$ RH. Three days after infestation, plants were used in the behavioural assay. Additionally, after each assay, P. xylostella caterpillars were removed from the plants and individually weighed on a microbalance (accuracy $1 \mu \mathrm{g}$; CP2P, Sartorius AG, Göttingen, Germany).

The behavioural responses of D. semiclausum to HIPV blends from Col-0 plants, tps03, tps 10 or bsmt1 mutant plants were investigated for the following comparisons: (a) P. xylostella-infested plants versus undamaged control plants 
were investigated to assess whether the parasitoid is attracted to volatiles induced by its host, (b) dual LD plants versus Dual HD plants to assess whether the parasitoid discriminates between HIPV emitted from plants with non-hosts at either high or low density. The effect of aphid infestation on the attraction to HIPVs from P. xylostella-infested plants was investigated by comparing the response to the HIPV blend of P. xylostella-infested Col-0 plants versus either Dual LD or Dual HD Col-0 plants.

\section{Gene expression analysis}

To link behavioural responses of D. semiclausum to the transcription of genes important for the biosynthesis of plant volatiles, we additionally performed a gene-expression analysis on Col-0 plants, tps 03 , tps 10 and bsmt 1 mutants that were used to assess parasitoid preference.

Before tissue collection, insects were removed from the plants with a fine brush. For each treatment, a total of eight leaves from four different plants used as odour source in the Y-tube behavioural assay were pooled to obtain one biological replicate. We selected fully expanded leaves that displayed insect feeding damage. In total, four biological replicates per genotype per treatment were used. Leaf tissue was snap-frozen in liquid nitrogen and stored at $-80{ }^{\circ} \mathrm{C}$ prior to analysis.

Finely ground, frozen plant leaf tissue was used for isolation of total RNA with the RNeasy Plant Mini Kit (Qiagen, Hilden, Germany). Total RNA samples were treated with DNase (Qiagen, Hilden, Germany). With the help of the iScript cDNA synthesis Kit (Bio-Rad), cDNA was synthesized from $1 \mu \mathrm{g}$ RNA. Quantitative RT-PCR analysis was performed in a CFX96 Touch ${ }^{\mathrm{TM}}$ Real-Time PCR Detection System (Bio-Rad). Each reaction was performed in a total volume of $25 \mu \mathrm{L}$ containing $12.5 \mu \mathrm{L}$ SYBR Green Supermix (Bio-Rad), $5 \mu \mathrm{L}$ cDNA and $1 \mu \mathrm{L}$ of $10 \mu \mathrm{M}$ forward and reverse gene-specific primer pair. For each reaction, two technical replicates were performed and average values were used in the analyses. The studied genes were the terpene synthase (TPS) genes TPSO3 (At4g16740), TPS04 (At1g61120) and TPS10 (At2g24210), the salicylic acid methyl transferase gene BSMT1 (At3g11480) and the two reference genes ELONGATION FACTOR $1 \alpha(E F 1 \alpha)$ (At5g60390) and GLYCERALDEHYDE-3-PHOSPHATE DEHYDROGENASE (GAPDH) (At3g04120). The following thermal profile was used for reactions with TPSO3, TPSO4 and BSMTI: 3 min $95^{\circ} \mathrm{C}$, followed by 40 cycles of $15 \mathrm{~s}$ at $95^{\circ} \mathrm{C}$, and $45 \mathrm{~s}$ at $60^{\circ} \mathrm{C}$. For reactions with TPS10 thermal conditions consisted of $3 \min 95^{\circ} \mathrm{C}$, followed by 40 cycles of $15 \mathrm{~s}$ at $95^{\circ} \mathrm{C}$, and $45 \mathrm{~s}$ at $62{ }^{\circ} \mathrm{C}$.

The two reference genes, GAPDH and EF1 $\alpha$, were carefully selected after evaluating their expression stability by calculating the geNorm value and coefficient of variation
(CV) (qbase+ v. 2.6.1, Biogazelle; Hellemans et al. 2007). Relative expression for each tested gene was calculated by using the geometric mean of threshold cycle $(\mathrm{Ct})$ values (Vandesompele et al. 2002) from the two reference genes with the $2^{-\Delta \Delta \mathrm{Ct}}$ method (Livak and Schmittgen 2001).

\section{Headspace collection}

Plant volatiles were collected from four A. thaliana Col-0 plants subjected to one of the four treatments as described in the 'Olfactory responses of $D$. semiclausum' section above. We used Col-0 plants as a marker to identify plant volatile profiles induced by multiple insect attacks. Future studies should explore the use of volatile-biosynthesis mutants for plant volatile collection to gain additional insight into plant responses to multiple herbivory.

For each treatment, seven replicates were sampled. The pots containing the four plants were carefully wrapped in aluminium foil and placed in a clean 5-L glass jar. The jars were sealed with a Viton-lined glass lid with an air inlet and outlet. Volatile control samples were collected from empty glass jars and from aluminium-wrapped pots filled with soil in order to correct for non-plant-related volatiles. Prior to volatile collection, the jars were ventilated for $30 \mathrm{~min}$ using charcoal-filtered compressed air. Plant volatiles were collected on 200 mg Tenax TA (20/35 mesh; CAMSCO, Houston, TX, USA) in a stainless steel cartridge by drawing air from the jars using an external pump at $200 \mathrm{~mL} \mathrm{~min}^{-1}$ for $6 \mathrm{~h}$, starting at $10 \mathrm{am}$. Immediately after each volatile collection, insects were removed from the plants and plant shoots of each treatment were pooled and weighed on an analytical balance (accuracy $0.1 \mathrm{mg}$; Mettler Toledo ML54/01). The Tenax TA cartridges were dry-purged under a stream of

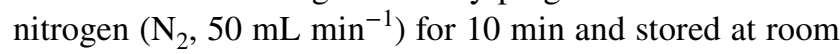
temperature $\left(22 \pm 2{ }^{\circ} \mathrm{C}\right)$ until analysis.

\section{Chemical analysis of volatiles}

Plant volatiles were identified and quantified as described by Pangesti et al. (2015). Separation and detection of plant volatiles was done using a Thermo Trace Ultra gas chromatograph (GC) coupled to a Thermo Trace DSQ quadrupole mass spectrometer (MS) (Thermo Fisher Scientific, Waltham, USA). The volatiles were thermally released from the Tenax TA cartridges at $250{ }^{\circ} \mathrm{C}$ for 10 min with a helium flow of $20 \mathrm{~mL} \mathrm{~min}{ }^{-1}$ on an Ultra 50:50 thermal desorption unit (Markes, Llantrisant, UK), while being focused on a cold sorbent trap at $0{ }^{\circ} \mathrm{C}$ (Unity, Markes). After completion of the desorption process, volatile compounds were released from the cold trap by ballistic heating at $40{ }^{\circ} \mathrm{C} \mathrm{s}^{-1}$ to $280^{\circ} \mathrm{C}$, which was maintained for $10 \mathrm{~min}$ and were then transferred in a splitless mode to an analytical column [(ZB$5 \mathrm{MSi} ; 30 \mathrm{~m} \times 0.25 \mathrm{~mm}$ i.d. $\times 0.25 \mu \mathrm{m}$ film thickness with 
$5 \mathrm{~m}$ built-in guard column (Phenomenex, Torrance, CA, USA)] situated inside the GC oven. The temperature of the GC oven was initially held at $40{ }^{\circ} \mathrm{C}$ for 2 min, which was then raised at $10{ }^{\circ} \mathrm{C} \mathrm{min}^{-1}$ to a final temperature of $280{ }^{\circ} \mathrm{C}$ and held for 4 min under a helium flow of $1 \mathrm{~mL} \mathrm{~min}^{-1}$. The DSQ MS was operated in a scan mode with $35-350 \mathrm{amu}$ mass range at 5.38 scans s $^{-1}$ and spectra were recorded in electron impact ionization (EI) mode at $70 \mathrm{eV}$. MS transfer line and ion source were set to 275 and $250{ }^{\circ} \mathrm{C}$, respectively. Volatile compounds were tentatively identified by comparison of mass spectra with those in the NIST 2005 and the Wageningen Mass Spectral Database of Natural Products MS libraries, as well as using experimentally obtained linear retention indices (LRI).

\section{Statistical analyses}

To determine whether parasitoid preferences and response rates differed between the various odour sources, data on olfactory responses of D. semiclausum were analysed using a $\chi^{2}$ test in SPSS v. 22.0 (SPSS Inc., Chicago, IL, USA) for each choice situation tested. In addition, data were analysed using a generalized linear model (GLM) with Poisson distribution and log link function in GenStat v. 17 (VSN International, Hemel Hempstead, UK) to compare choice distributions between plant genotypes. Genotype and treatment combination (i.e. undamaged plants tested in the Y-tube olfactometer against $P$. xylostella-damaged plants or plants infested by both $P$. xylostella and a low density of five aphids per plant (hereafter abbreviated as Dual LD for dual low density) tested against plants infested by both $P$. xylostella and a high density of 25 aphids per plant [abbreviated as Dual HD for dual high density)] and the interaction genotype $\times$ treatment combination were included as fixed factors for data on proportion of responsive or non-responsive wasps. In the choice assays involving undamaged plants, the number of wasps choosing the P. xylostella-infested plants out of the total number of responding wasps was entered as the response variable. In the choice assays between Dual LD versus Dual HD, the number of wasps choosing the Dual HD plants out of the total number of responding wasps was entered as the response variable. The dispersion parameter was estimated to account for residual variance. Post hoc comparisons for proportion of responsive or non-responsive wasps were analysed with a least significant difference (LSD) test.

Fisher's exact test (two-tailed) was used to determine whether parasitoid preferences were distributed identically across different days on which the tests were repeated. After each behavioural bioassay, we tested if there were differences in weight of $P$. xylostella caterpillars feeding alone or simultaneously with aphids at low or high density on plants. Data of P. xylostella larval weight were analysed with a linear mixed model with treatment as fixed factor and experimental group (i.e. the four A. thaliana plants subjected to one of treatments used in the behavioural bioassay) as random factor. Effect of treatment on plant shoot fresh weight was analysed with an independent samples $t$ test. The statistical analysis of $P$. xylostella larval weight and plant shoot weight was carried out using SPSS v. 22.0 (SPSS Inc., Chicago, IL, USA).

The expression of genes and the quantity of each volatile emitted by plants on which caterpillars were feeding alone or simultaneously with aphids at either density or left undamaged were compared using a GLM with Poisson distribution and log link function in GenStat v. 17.0 (VSN International, Hemel Hempstead, UK). The factor treatment was included in the model as fixed factor. The dispersion parameter was estimated to account for residual variance. Post hoc comparisons for gene expression and volatile data were analysed with an LSD test. Data on volatile emission were also investigated by discriminant analysis. The quantified peak areas of individual volatile compounds were divided by plant shoot fresh mass, log-transformed, univariate scaled and mean-centred prior to subjecting the data to a multivariate data analysis: orthogonal projection to latent structures discriminant analysis (OPLS-DA) using SIMCA-P+ version 14.0 statistical software (Umetrics AB, Umeå, Sweden). The analysis determines whether samples from different treatment groups can be separated on the basis of quantitative and qualitative differences in their volatile blends. The results of the analysis are visualized in score and loading plots. The score plot identifies patterns that discriminate between the sample groups according to the two given model components of OPLS-DA, i.e. the predictive and orthogonal component. The predictive component corresponds to variation between the sample treatments, whereas the orthogonal component corresponds to withinsample variation. The loading plot displays the contribution and variable importance in the projection (VIP) of each volatile compound for the discrimination between the sample groups. Volatile compounds with VIP $>1$ are considered most influential in the model (Eriksson et al. 2013). Pairwise OPLS-DA analyses were conducted on the volatile blends of the different treatment groups. The quality of each OPLS-DA model was evaluated using the parameter $R^{2} X$, which is used to assess the stability of the model (providing a quantitative measure of the explained variation) and indicates goodness of fit (Eriksson et al. 2013). 


\section{Results}

\section{Olfactory responses of the caterpillar parasitoid Diadegma semiclausum to HIPV blends}

Parasitoid preference was studied for caterpillar-infested versus uninfested Col- 0 plants and mutants impaired in the biosynthesis of linalool (synthesized by TPS10), MeSA (synthesized by BSMT1) and $(E, E)-\alpha$-farnesene (synthesized by TPSO3). Female D. semiclausum parasitoids preferred volatiles emitted by $P$. xylostella-infested plants of Col-0 wild type, as well as tps03 and bsmt 1 mutants over those from undamaged Col-0, tps03 or bsmt1 plants (Fig. 1a; $\chi^{2}$ test, Col-0: $\chi^{2}=4.261, P=0.039$; tps03: $\chi^{2}=5.828$, $P=0.016$; bsmt $\left.: \chi^{2}=5.769, P=0.016\right)$. Parasitoids did not discriminate between $P$. xylostella-infested tps 10 mutants and undamaged tps 10 mutants (Fig. $1 \mathrm{a} ; \chi^{2}$ test, $\chi^{2}=2.462$, $P=0.12)$.

When comparing parasitoid responses to dually infested plants with either a low or high aphid density, the parasitoids discriminated between volatiles from Dual LD and Dual HD plants for the Col-0 plants and tps03 mutant. The parasitoids preferred the volatile blend from Dual HD over volatiles emitted by Dual LD plants for Col-0 (Fig. 1b; $\chi^{2}$ test, $\chi^{2}=5.233, P=0.022$ ), whereas the opposite was recorded for tps03 plants: parasitoids significantly preferred the volatile blend from Dual LD tps03 mutants over those from Dual HD tps03 mutants (Fig. $1 \mathrm{~b} ; \chi^{2}$ test, $\chi^{2}=5.121$, $P=0.024)$. The wasps did not discriminate between the volatile blend from Dual LD or Dual HD tps 10 and bsmt 1 mutants (Fig. $1 \mathrm{~b} ; \chi^{2}$ test, tps $10 ; \chi^{2}=0.641, P=0.42$; bsmtl: $\left.\chi^{2}=0.714, P=0.40\right)$.

The presence of $B$. brassicae at low or high density did not interfere with $D$. semiclausum's response to volatiles from $P$. xylostella-infested plants: parasitoids did not discriminate between the volatile blend from Col-0 plants infested by caterpillars and the volatile blend from Dual LD or Dual HD Col-0 plants (Fig. 1c; $\chi^{2}$ test, Dual LD: $\chi^{2}=0.364, P=0.55$; Dual HD: $\left.\chi^{2}=0.022, P=0.88\right)$.

To further investigate the role of linalool, MeSA and $(E, E)$ - $\alpha$-farnesene in mediating parasitoid preference and responsiveness, we compared parasitoid behaviour in response to herbivore-infested Col-0 plants to those in response to herbivore-infested volatile-biosynthesis mutants. Parasitoid preference was influenced by the different genotypes and treatment combinations tested (Online Resource 6; GLM). There were no significant differences between the different genotypes for the proportion of parasitoids that preferred volatiles from $P$. xylostella-infested plants over those from uninfested plants (Fig. 1a). Interestingly, volatiles emitted by Dual HD tps03 mutants significantly affected parasitoid preference compared to Dual HD Col-0 plants (Fig. 1b). There were no significant differences for

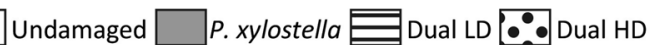

a

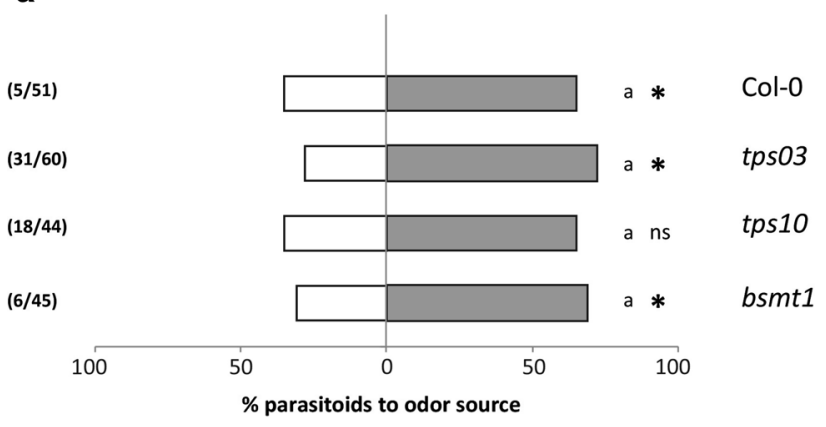

b
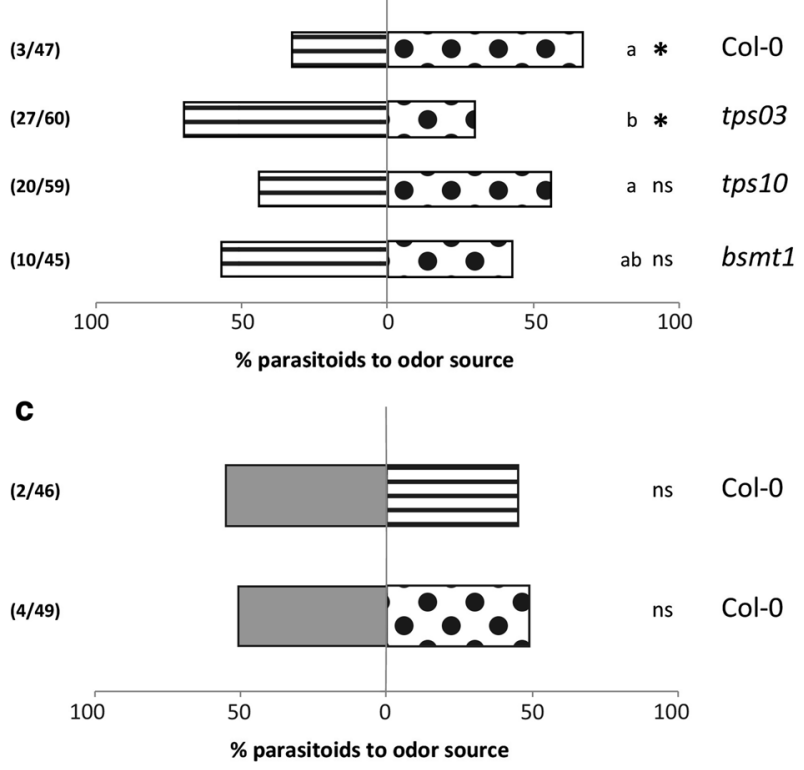

Fig. 1 Preference of D. semiclausum in a Y-tube olfactometer to volatile blends emitted by Arabidopsis Col-0 wild type, tps03, tps10 or bsmt 1 mutants after three days of insect infestation. Undamaged plants were tested against plants infested by two L2 P. xylostella caterpillars (a), plants dually infested by caterpillars and a low density of five $B$. brassicae aphids (Dual LD) were tested against plants dually infested by caterpillars and a high density of $25 \mathrm{~B}$. brassicae aphids (Dual HD) (b), or Col-0 plants infested by caterpillars were tested against Col-0 plants dually infested by $P$. xylostella caterpillars and a low aphid density (c), or Col-0 plants infested by caterpillars were tested against plants dually infested by $P$. xylostella caterpillars and aphids at a high density (c). Each bar represents the percentage of wasps choosing for each of the two odour sources, which consisted of four plants per treatment. For each pair-wise comparison, 3-4 sets of plants were tested on different days. An asterisk indicates a significant preference within a dual-choice test: $n s$ not significant; asterisk, $P<0.05\left(\chi^{2}\right.$ test $)$. Parasitoid preference that is significantly different between the different genotypes is indicated with different letters (GLM, $P<0.05$ ). Numbers in parentheses represent number of nonresponsive wasps and total number of tested wasps, respectively

parasitoid preference in response to Dual LD and Dual HD Col-0 plants and to the same treatment combination for tps 10 and bsmtl mutants (Fig. 1b). 
Analysis of the response of wasps towards volatile blends emitted by either Col-0 plants or volatile-biosynthesis mutants, showed an effect of plant genotype but no effect of the treatment combinations offered (i.e. undamaged versus $P$. xylostella or Dual LD versus Dual HD) (Online Resource 7; GLM). Volatiles emitted by tps 10, tps03 and bsmt1 mutants increased the percentage of wasps that did not make a choice compared to Col-0 plants.

Thus, blocking the biosynthesis of linalool, $(E, E)-\alpha-$ farnesene and MeSA, does not influence preference of $D$. semiclausum parasitoids for plants infested by P. xylostella caterpillars versus uninfested plants. On the other hand, mutations in TPSO3, TPS10 and BSMT1 reduce the responsiveness of the wasps. In addition, $(E, E)-\alpha$-farnesene is required for the density-dependent effect on attraction of parasitoids to plants infested by both caterpillars and aphids.

Preference of $D$. semiclausum parasitoids was not influenced by the day on which the experiments were performed for Col-0 plants and tps10, bsmt1 or tps03 mutants (Fisher's exact test, $P>0.05)$. Furthermore, caterpillar body mass reached similar values when feeding on Col-0 plants, tps 10 , bsmt1 or tps03 mutants (Online Resource 1; LMM, $P>0.1$ ) tested during the Y-tube olfactometer bioassays.

\section{Plant volatile emission}

Emission of volatiles was analysed to study if differences in volatile profile between plant treatments could explain the observed differences in parasitoid preference.

In total, 41 different volatile compounds were detected in the headspace of all treatments (Online Resource 4). An OPLS-DA model comparing headspace samples from all four treatments showed differences in volatile blends based on the presence or absence of herbivores. The first two components of the OPLS-DA, i.e. the predictive and orthogonal component, are plotted in the model (Fig. 2a). The predictive component explained $13.29 \%$ of the variance, while $20.15 \%$ was explained by the first of two orthogonal components.
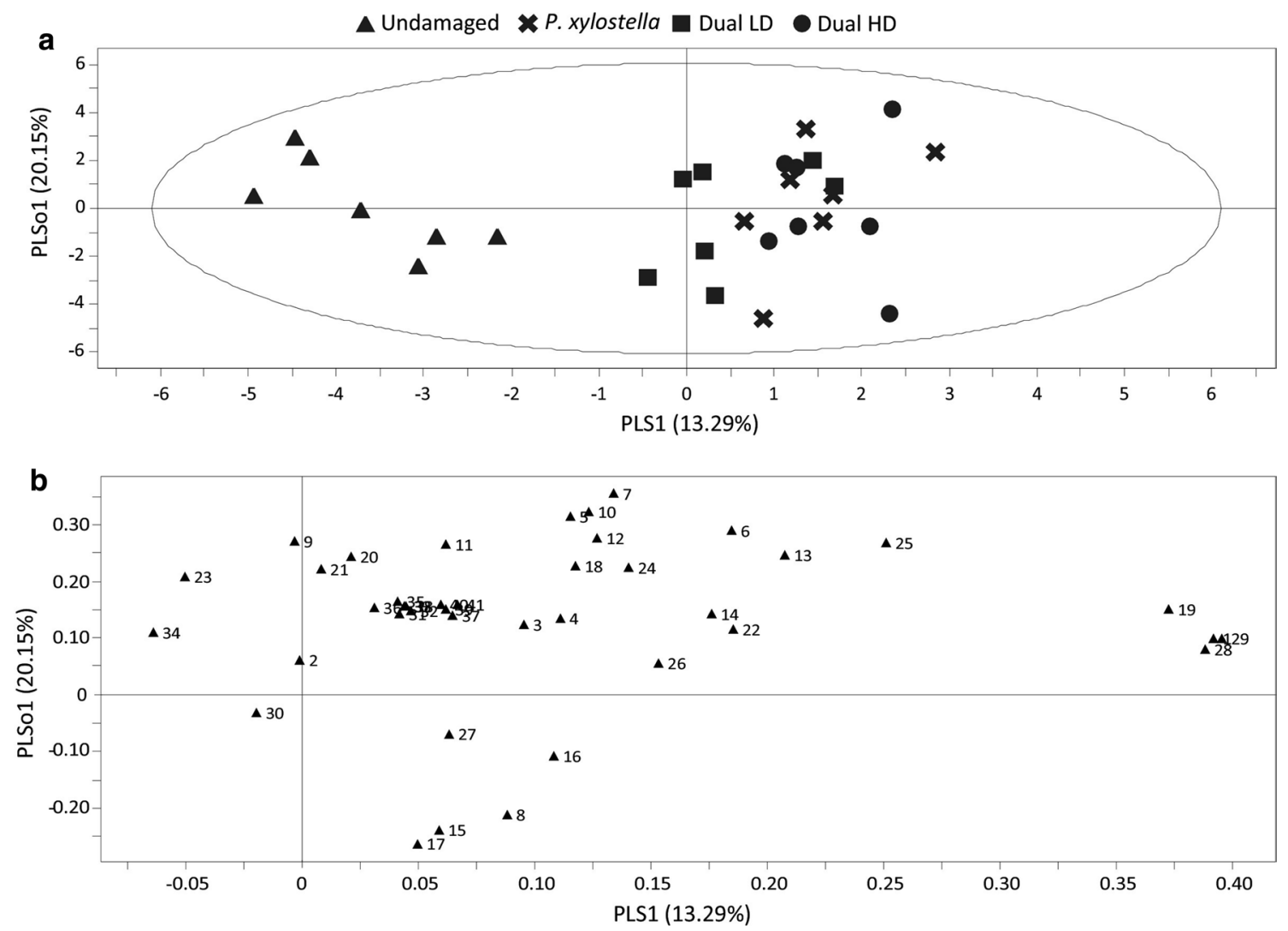

Fig. 2 Orthogonal projection to latent structures discriminant analysis (OPLS-DA) of volatile compounds emitted by Arabidopsis wild-type Col-0 plants after 3 days of insect infestation. Plants were infested by $P$. xylostella alone, dually infested by $P$. xylostella and $B$. brassicae (Dual) or left undamaged. Plants were infested with either a low (LD) or a high (HD) density of B. brassicae aphids. a Score plot displaying grouping pattern according to the first two model components and the Hotelling's ellipse of the $95 \%$ confidence interval for the observations. Each point represents one sample ( $n=7$ replicates). The OPLS-DA resulted in a model with one significant predictive and two significant orthogonal components with $R^{2} X=0.639$. b Loading plot of the first two components of OPLS-DA, showing the contribution of each volatile compound to the separation of the four treatments. Numbers refer to the volatile compounds listed in Online Resource 4 
A group of eight compounds contributed most strongly to the model (VIP > 1), indicating that these compounds contributed most to the difference between the volatile blends (Online Resource 5). Based on the three highest VIP-values, 1-penten-3-ol, $(E, E)$-TMTT and $(E, E)$ - $\alpha$-farnesene influenced the separation of undamaged and herbivore-infested plants the most (Fig. 2b). These three compounds were emitted in significantly higher amounts by herbivore-infested plants than by undamaged plants (Online Resource 4).

Pair-wise comparison by OPLS-DA for volatiles emitted by undamaged plants and plants infested by P. xylostella caterpillars shows a clear separation based on the presence or absence of $P$. xylostella caterpillars. The first two components of the OPLS-DA are plotted in the model (Fig. 3a). The predictive component explained $16.06 \%$ of the variability, while $20.62 \%$ was explained by the first of seven orthogonal components. A group of 12 plant volatile compounds contributed most strongly to the model (VIP > 1), indicating that these compounds contributed to the difference between the volatile blends (Online Resource 5). Based on the four highest VIP-values,
$(E, E)$-TMTT, 1-penten-3-ol, $(E, E)$ - $\alpha$-farnesene and MeSA influenced the separation of volatile blends from undamaged and caterpillar-infested plants the most (Fig. 3b). These four compounds were emitted in significantly higher amounts by caterpillar-infested plants than by undamaged plants (Online Resource 4).

An OPLS-DA model including volatiles emitted by Dual LD plants and Dual HD plants showed a clear separation between the two treatments. The first two components of the OPLS-DA model are plotted in the model (Fig. 4a) and explain $34.53 \%$ of the total variance. A group of 13 plant volatile compounds contributed most strongly to the model (VIP > 1) (Online Resource 5). Based on the three highest VIP-values, 1-penten-3-ol, $(E, E)$ - $\alpha$-farnesene and linalool influenced the separation of the two treatments the most (Fig. 4b).

Volatile blends emitted by $P$. xylostella-infested plants and Dual LD or Dual HD plants were not separated by OPLS-DA.

After each collection of volatile compounds plant shoot fresh weight was measured. There was no effect of insect

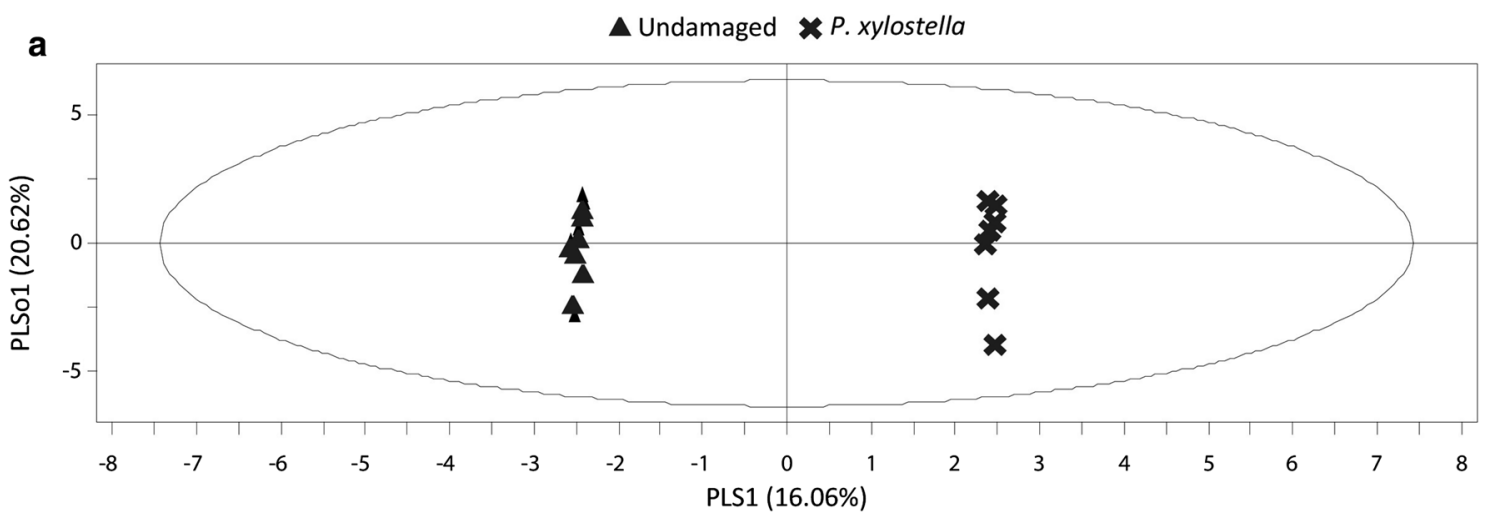

b

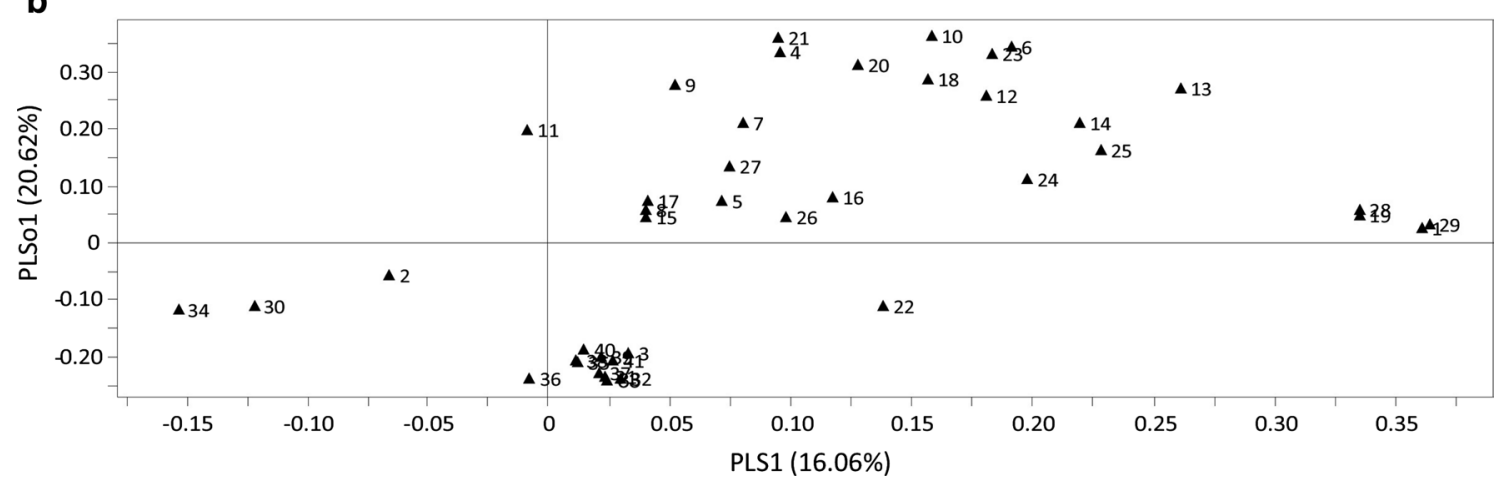

Fig. 3 Orthogonal projection to latent structures discriminant analysis (OPLS-DA) of volatile compounds emitted by Arabidopsis wild-type Col-0 plants after 3 days of insect infestation. Plants were infested by $P$. xylostella caterpillars or left undamaged. a Score plot displaying grouping pattern of samples according to the first two model components and the Hotelling's ellipse of the 95\% confidence interval for the observations. Each point represents one sample
( $n=7$ replicates). The OPLS-DA resulted in a model with one significant predictive and seven significant orthogonal components with $R^{2} X=0.908$. b Loading plot of the first two components of OPLSDA, showing contribution of each volatile compound to the separation of the two treatments. Numbers refer to the volatile compounds listed in Online Resource 4 

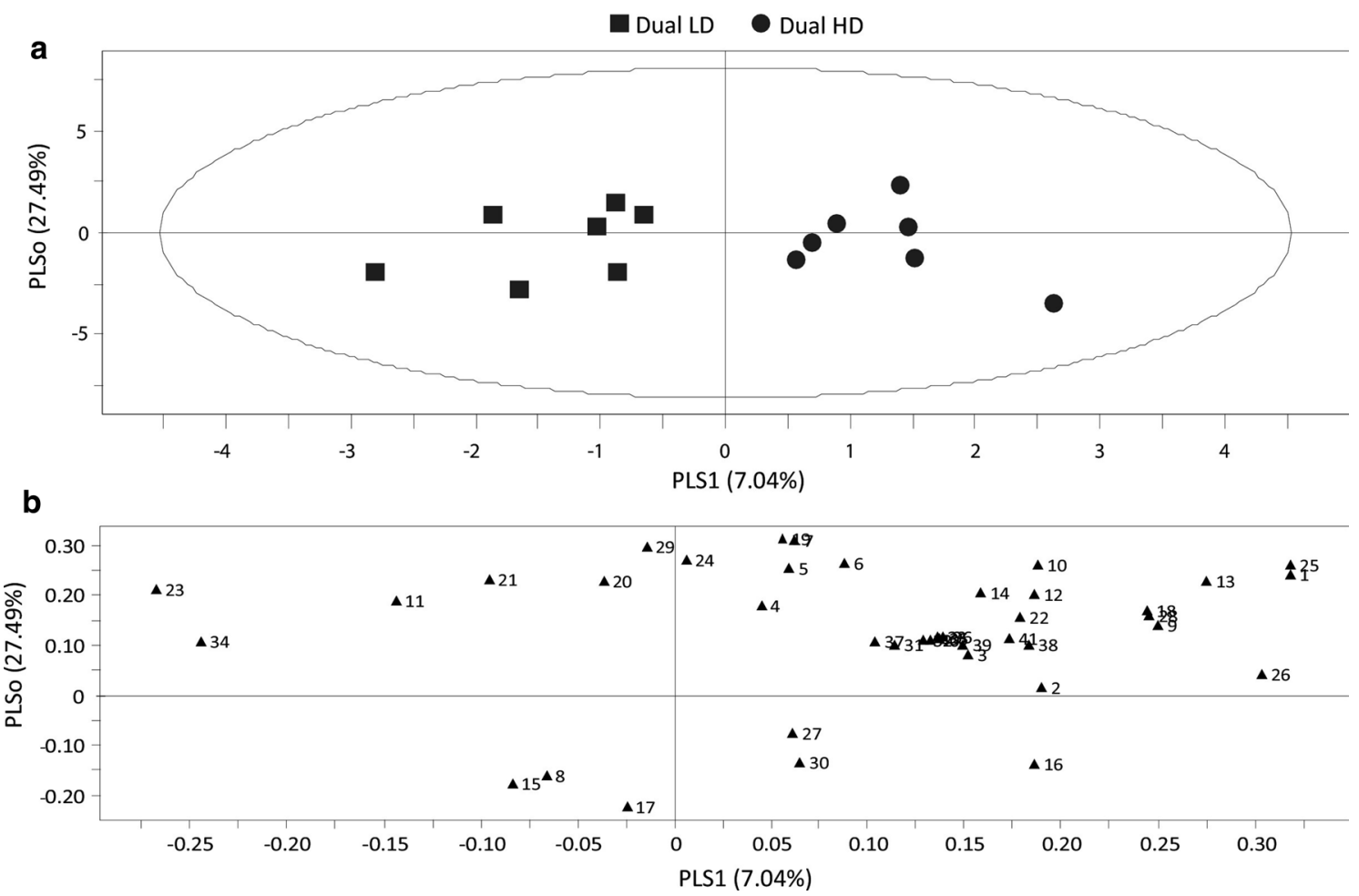

Fig. 4 Orthogonal projection to latent structures discriminant analysis (OPLS-DA) of volatile compounds emitted by Arabidopsis wildtype Col-0 plants after 3 days of insect infestation. Plants were dually infested by $P$. xylostella and a low B. brassicae density (Dual LD, 5 aphids) or by $P$. xylostella and a high B. brassicae density (Dual $\mathrm{HD}, 25$ aphids). a Score plot displaying grouping pattern according to the first two model components and the Hotelling's ellipse of the

infestation on plant biomass (Online Resource 2; ANOVA, $P>0.1)$.

\section{Transcriptional analysis of TPS03, TPS04, TPS10 and BMST1}

To explain the observed HIPV-profiles, transcript levels of genes important for their biosynthesis (i.e. TPS03, TPSO4 and TPS10 in the terpenoid biosynthesis pathway, and BSMT1 in the methyl salicylate biosynthesis pathway) were analysed in Col-0 plants and tps 10, bsmt1 and tps03 mutants used in the Y-tube behavioural bioassays.

Expression of TPSO3, TPS10 and BSMT1 was verified in the mutants tps03, tps 10 and bsmt1, respectively. Caterpillar-induced expression of TPSO3, TPS10 and BSMT1 was severely reduced in their corresponding mutants when compared with Col-0 wild-type plants (Online Resource 3; GLM, $P<0.02)$.

There was a significant effect of treatment on the expression of TPSO3 and TPS10 in Col-0 plants, of TPSO3 and BSMT1 in tps 10 mutants, of TPS10 in bsmt 1 mutants and of TPSO4, TPS10 and BSMT1 in tps03 mutants (Online
95\% confidence interval for the observations. Each point represents one sample ( $n=7$ replicates). The OPLS-DA resulted in a model with one significant predictive and two significant orthogonal components with $R^{2} X=0.620$. b Loading plot of the first two components of OPLS-DA, showing contribution of each volatile compound to the separation of the two treatments. Numbers refer to the volatile compounds listed in Online Resource 4

Resource 8). However, due to variation in BSMT1 expression level within treatment type, no significant differences between treatments for BSMT1 expression level were found for Col-0 plants and tps 10 mutants (Fig. 5).

In Col-0 plants, feeding by caterpillars plus aphids at a high density induced TPSO3 expression to a higher level compared to simultaneous feeding of caterpillars and aphids at low density (Fig. 5a).

This shows that aphids influence TPSO3 expression level in a density-dependent manner. Interestingly, no significant difference was found between TPSO3 expression levels in tps 10 and bsmt 1 mutants infested by caterpillars plus a low or high aphid density (Fig. 5a). Furthermore, expression levels of TPSO3 in P. xylostella-infested tps 10 and $b s m t 1$ mutants remained unchanged compared to uninfested (Control) plants (Fig. 5a).

Feeding by $P$. xylostella caterpillars, regardless of whether aphids were present as well, induced the expression of genes important for the biosynthesis of volatiles in plants. Significantly higher expression levels of TPSO4 in tps03 mutants, of TPS10 in Col-0 and bsmt1 plants, and 

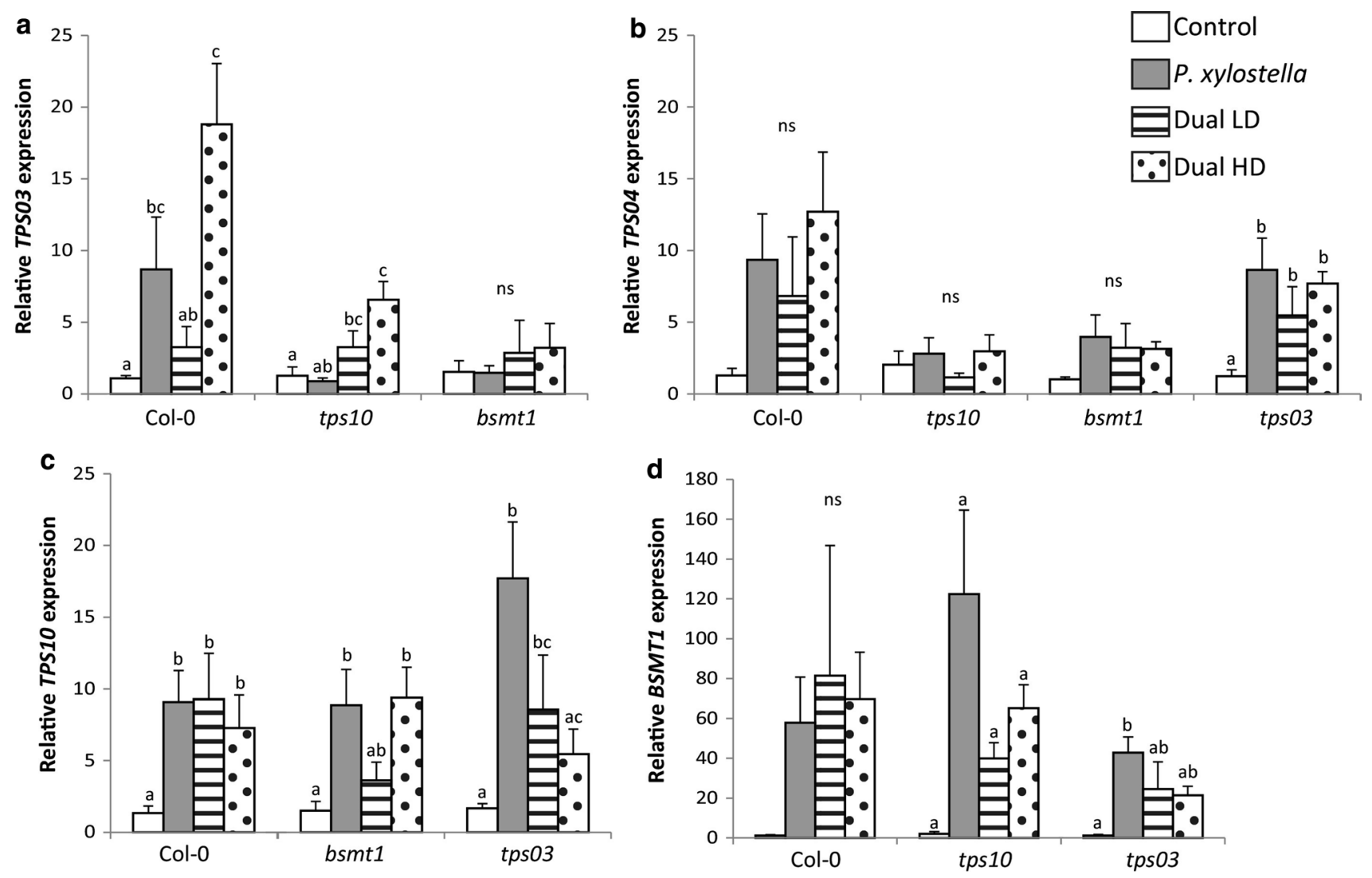

Fig. 5 Gene expression in leaves of A. thaliana wild-type Col-0 and mutants tps10, bsmt 1 and tps03 used during the Y-tube olfactometer bioassays after single $P$. xylostella and dual P. xylostella and B. brassicae infestation (Dual) at either a low (LD, 5 aphids) or high (HD,

25 aphids) aphid density and without infestation (control). Bars represent mean $\pm \operatorname{SE}$ ( $n=4$ biological replications). Bars marked with different letters are significantly different between treatments (GLM, $P<0.05 ;$ ns not significant)

of BSMT1 in tps03 mutants were found upon caterpillar feeding compared to control plants (Fig. 5).

In tps03 mutants, caterpillars feeding alone and simultaneous feeding by caterpillars and aphids at low density induced TPS10 expression to a significantly higher level compared to uninfested plants (Fig. 5). TPS10 expression was negatively correlated with aphid density on caterpillarinfested tps03 plants (Fig. 5). Expression of TPS10 is significantly affected by feeding of caterpillars in combination with aphids at high density in tps03 mutants compared to control plants. Caterpillars and aphids at high density feeding on tps03 mutants induced significantly lower levels of TPS10 expression compared to caterpillars feeding alone, which was not found for Col-0 plants (Fig. 5). This indicates that aphids at high density feeding simultaneously with caterpillars interfere with caterpillar-induced TPS10 expression.

\section{Discussion}

We investigated the effect of simultaneous feeding by $B$. brassicae aphids and P. xylostella caterpillars on induced

plant responses and the attraction of the parasitoid $D$. semiclausum, an important natural enemy of $P$. xylostella caterpillars. Our study shows that $D$. semiclausum parasitoid attraction is influenced by the density of the aphids. Parasitoids preferred the volatile blend of dually infested plants at high aphid density over those from dually infested plants at low aphid density. No discrimination was recorded when host-infested plants were offered versus host-infested plants with either a low or a high aphid density. This suggests that different aphid densities have opposing effects, which is supported by our previous work on the effects of high and low aphid densities on P. xylostella (Kroes et al. 2015).

It has been observed before that the level of induced indirect plant defence is influenced by the density of the herbivores feeding on the plant (Dudareva et al. 2006). For instance, attraction of the predatory mite $P$. persimilis to volatiles from lima bean plants (Phaseolus lunatus) infested by spider mites ( $T$. urticae) and attraction of the parasitoid Cotesia vestalis to volatile blends emitted by P. xylostellainfested cabbage plants (B. oleracea) are positively densitydependent (Gols et al. 2003; Girling et al. 2011). 
Compared with feeding by only one insect species, herbivory by a second herbivore may influence indirect defence responses (Rodriguez-Saona et al. 2003, 2005; Heil 2008; Dicke et al. 2009; Erb et al. 2010; Zhang et al. 2013; Ponzio et al. 2016), which can also be affected by the density of the attacking insects (Zhang et al. 2009; Kroes et al. 2015). Simultaneous feeding by phloem-feeding whiteflies and Spodoptera exigua caterpillars on cotton plants (Gossypium hirsutum) reduced the emission of DMNT [ $(E)$ 4,-8-dimethyl-1,3,7-nonatriene], TMTT and the monoterpene myrcene compared to plants infested by only $S$. exigua (Rodriguez-Saona et al. 2003). Zhang et al. (2013) showed that feeding by $B$. tabaci whiteflies significantly reduced the attraction of $D$. semiclausum parasitoids to volatile blends from A. thaliana plants simultaneously infested by P. xylostella, which was associated with differences in the HIPV blend. Our present results show that feeding by B. brassicae aphids does not affect the preference of $D$. semiclausum for P. xylostella-infested plants over control uninfested plants. This indicates that aphids and whiteflies, although both phloem feeders, differentially induce plant responses. Our data indicate that feeding by caterpillars plus aphids at a low density suppressed transcription of TPSO3 (encoding an $(E, E)$ - $\alpha$-farnesene synthase) compared to simultaneous feeding by caterpillars and aphids at a high density. This indicates that TPSO3 expression in response to both caterpillar and aphid feeding depends on aphid density. In addition, olfactory responses of $D$. semiclausum to volatiles emitted by dual-infested tpsO3 mutants confirmed that a functional TPSO3 in A. thaliana is required for interference by aphids.

Results from the volatile analysis show that the volatile blends changed depending on simultaneous feeding by caterpillars and aphids at low or high density and this may explain the behavioural responses by $D$. semiclausum. Whether the responses benefit the parasitoids in terms of offspring performance remains to be investigated. Such performance effects may be present in terms of, e.g. offspring development rate and level of host immunity (Rodriguez-Saona et al. 2005; Bukovinszky et al. 2009; Soler et al. 2012).

In the headspace of plants infested by caterpillars alone, dually infested by caterpillars and aphids at both densities or undamaged plants, the same compounds were detected. Moreover, four of these compounds (1-penten-3-ol, $(E, E)$ TMTT, $(E, E)-\alpha$-farnesene and linalool) were found to be important for the separation of the different blends in the multivariate data analysis. Thus, this study underlines the significance of the quantitative composition of volatile blends used by parasitoids to locate host-infested plants. However, it is noteworthy that parasitoids are able to detect very subtle differences in volatile blends which are difficult to identify by chemical analysis (Clavijo McCormick et al. 2014; Ponzio et al. 2016), leaving the possibility open that other HIPVs contributed to the discrimination exhibited by the parasitoids. In addition, no difference in $(E, E)-\alpha-$ farnesene emission by dual-infested plants at low density and high density were found, whereas transcript levels of the corresponding TPSO3 gene did differ between treatments. This may be related to substrate availability (Degenhardt et al. 2009; Tholl and Lee 2011) or through posttranslational protein modifications (Tholl et al. 2005). Furthermore, sesquiterpenes, such as $(E, E)-\alpha$-farnesene, are known to be unstable volatile compounds that are rapidly oxidized (Anet $1969)$, which may explain why $(E, E)-\alpha$-farnesene emission did not differ between dually infested Col-0 plants at low or high aphid density.

Phytohormonal crosstalk between JA- and SA-mediated signalling pathways is thought to underlie plant-mediated interactions with multiple insect species and behavioural responses of parasitoids and predators (Zhang et al. 2013; Stam et al. 2014; Wei et al. 2014). Activation of SA-signalling in response to aphid feeding (Moran et al. 2002; Mewis et al. 2006; Kusnierczyk et al. 2011) may suppress JA-dependent indirect defence responses. This may result in changes in the composition of the volatile blend (Truong et al. 2014). On the other hand, we found that feeding by $P$. xylostella caterpillars alone induced not only JA-regulated terpenoid volatiles in A. thaliana but, similar to the finding of Zhang et al. (2013), also relatively high levels of MeSA, the methyl ester of SA. This indicates that the general pattern of negative crosstalk between SA- and JAdependent signalling pathways in the interactions between simultaneous feeding caterpillars and aphids does not always apply. Interestingly, the data show that mutation in BSMT1 (that catalyses the synthesis of MeSA from SA) interfered with the responsiveness of D. semiclausum to host-infested plants. Similarly, it was shown by Snoeren et al. (2010) that the attraction of $D$. semiclausum was negatively affected by MeSA.

Induction of linalool and 1-penten-3-ol emission depends on the JA-signalling pathway (Fisher et al. 2003; Van Schie et al. 2007; Snoeren et al. 2010), whereas emission of these plant volatiles in response to caterpillar feeding was not affected by simultaneous $B$. brassicae feeding. Since it is known that the emission pattern of HIPVs varies over time and a time lag occurs between gene induction and subsequent volatile emission (Dudareva et al. 2006; Heil 2008), effects on HIPV emission by simultaneous feeding by caterpillars and aphids might have been found at other time points after induction. For example, an increase in the amount of emitted volatiles was found after $48 \mathrm{~h}$ in A. thaliana plants simultaneously infested by $P$. xylostella caterpillars and whiteflies compared to plants infested by caterpillars alone (Zhang et al. 2013).

In line with Houshyani et al. (2013) and Zhang et al. (2013), we also observed preference of D. semiclausum parasitoids for volatile blends from P. xylostella-infested $A$. 
thaliana plants. Mutations in the biosynthesis of linalool (tps10 mutants) could have modified the volatile blend after infestation by $P$. xylostella caterpillars and these changes reduced the attraction of $D$. semiclausum parasitoids to host-infested plants. Therefore, linalool may function as $D$. semiclausum attractant. Linalool has been reported before as an important attractant for D. semiclausum parasitoids (Houshyani et al. 2013) and has been found in P. xylostellainduced volatile blends from A. thaliana (Zhang et al. 2013; Online Resource 4). Other volatile compounds induced most strongly by feeding of $P$. xylostella caterpillars were myrcene and TMTT and, therefore, may contribute to the attraction of D. semiclausum parasitoids (Online Resource 4). These two specific compounds were also found in the volatile blend emitted by A. thaliana plants in response to P. xylostella infestation after 2 days of feeding (Zhang et al. 2013).

In conclusion, we have shown that the behavioural response of parasitoids to HIPVs emitted by plants dually attacked by aphids and caterpillars depends on aphid density and found changes in the HIPV blend associated with density. Biosynthesis and emission of $(E, E)$ - $\alpha$-farnesene were linked to the observed preference of $D$. semiclausum parasitoids for volatiles emitted by plants dually infested by caterpillars and aphids at a high density. In addition, biosynthesis of linalool and $(E, E)-\alpha$-farnesene strongly influenced $D$. semiclausum responsiveness to host-infested plants. Parasitoids are enemies of herbivorous insects that are important members of plant-associated communities. Here, we have investigated the effects of non-host herbivory on foraging behaviour of naïve parasitoids to assess effects early in parasitoid life. Parasitoids are well known to associatively learn environmental cues (Turlings et al. 1993; Hoedjes et al. 2011). Thus, they may also learn to associate cues related to encounters with hosts in multi-herbivore infestations. First evidence indicates that foraging by the parasitoid Cotesia glomerata is indeed influenced by previous oviposition experiences on plants infested by not only their hosts but also non-host herbivores (de Rijk 2016). As plants growing under field conditions are commonly attacked by multiple insect herbivores at the same time, a better understanding of how plants regulate indirect defence mechanisms in response to multiple insect attack and how parasitoids deal with plantproduced information will provide important knowledge on plant-mediated ecological interactions within plant-associated communities. Such multi-species interactions may be complex in themselves. Yet, the fact that non-host herbivore densities modulate these species interactions presents an additional layer of complexity.

Acknowledgements We thank Léon Westerd, Frans van Aggelen, André Gidding and Joop Woelke for culturing the insects. We would also like to thank Thierry Delatte and Dorothea Tholl for providing seeds of the mutants tps 10 and tps03.
Author contribution statement $\mathrm{MD}, \mathrm{JvL}$ and $\mathrm{AK}$ designed the research. AK and FC performed the research. AK and BW analysed data. AK, JvL and MD wrote the paper with input from BW and FC.

Compliance with ethical standards

Funding This study was funded by a TOP GO Grant (Grant number 854.10.010 to MD) from The Netherlands Organization for Scientific Research (NWO).

Conflict of interest The authors declare that they have no conflict of interest.

Open Access This article is distributed under the terms of the Creative Commons Attribution 4.0 International License (http://creativecommons.org/licenses/by/4.0/), which permits unrestricted use, distribution, and reproduction in any medium, provided you give appropriate credit to the original author(s) and the source, provide a link to the Creative Commons license, and indicate if changes were made.

\section{References}

Aharoni A, Giri AP, Deuerlein S, Griepink F, de Kogel WJ, Verstappen FW, Verhoeven HA, Jongsma MA, Schwab W, Bouwmeester HJ (2003) Terpenoid metabolism in wild-type and transgenic Arabidopsis plants. Plant Cell 15:2866-2884

Ali JG, Agrawal AA (2012) Specialist versus generalist insect herbivores and plant defence. Trends Plant Sci 17:293-302

Anet EFLJ (1969) Autoxidation of $\alpha$-Farnesene. Aust J Chem 22:2403-2410

Arimura G, Kost C, Boland W (2005) Herbivore-induced, indirect plant defences. Biochim Biophys Acta 1734:91-111

Aubourg S, Lecharny A, Bohlmann J (2002) Genomic analysis of the terpenoid synthase (AtTPS) gene family of Arabidopsis thaliana. Mol Genet Genom 267:730-745

Bukovinszky T, Poelman EH, Gols R, Prekatsakis G, Vet LEM, Harvey JA, Dicke M (2009) Consequences of constitutive and induced variation in plant nutritional quality for immune defence of a herbivore against parasitism. Oecologia 160:299-308

Cai X-M, Sun X-L, Dong W-X, Wang G-C, Chen Z-M (2013) Herbivore species, infestation time, and herbivore density affect induced volatiles in tea plants. Chemoecology 24:1-14

Chen F, D'Auria JC, Tholl D, Ross JR, Gershenzon J, Noel JP, Pichersky E (2003) An Arabidopsis thaliana gene for methylsalicylate biosynthesis, identified by a biochemical genomics approach, has a role in defence. Plant J 36:577-588

Clavijo McCormick A, Unsicker SB, Gershenzon J (2012) The specificity of herbivore-induced plant volatiles in attracting herbivore enemies. Trends Plant Sci 17:303-310

Clavijo McCormick A, Irmisch S, Reinecke A, Boeckler GA, Veit D, Reichelt M, Hansson BS, Gershenzon J, Kollner TG, Unsicker SB (2014) Herbivore-induced volatile emission in black poplar: regulation and role in attracting herbivore enemies. Plant Cell Environ 37:1909-1923

De Boer JG, Posthumus MA, Dicke M (2004) Identification of volatiles that are used in discrimination between plants infested with prey or nonprey herbivores by a predatory mite. J Chem Ecol 30:2215-2230

De Rijk M (2016) Foraging behaviour of parasitoids in multi-herbivore communities. PhD thesis Wageningen University, Wageningen, The Netherlands, p 218 
De Rijk M, Dicke M, Poelman EH (2013) Foraging behaviour by parasitoids in multiherbivore communities. Anim Behav $85: 1517-1528$

De Vos M, Van Oosten VR, Van Poecke RMP, Van Pelt JA, Pozo MJ, Mueller MJ, Buchala AJ, Métraux J-P, Van Loon LC, Dicke M, Pieterse CMJ (2005) Signal signature and transcriptome changes of Arabidopsis during pathogen and insect attack. Mol Plant Microbe Int 18:923-937

Degenhardt J, Kollner TG, Gershenzon J (2009) Monoterpene and sesquiterpene synthases and the origin of terpene skeletal diversity in plants. Phytochem 70:1621-1637

Dicke M, Baldwin IT (2010) The evolutionary context for herbivoreinduced plant volatiles: beyond the 'cry for help'. Trends Plant Sci 15:167-175

Dicke M, Van Poecke RMP (2002) Signalling in plant-insect interactions: signal transduction in direct and indirect plant defence. In: Scheel D, Wasternack C (eds) Plant signal transduction. Oxford University Press, Oxford, pp 289-316

Dicke M, Van Poecke RMP, De Boer JG (2003) Inducible indirect defence of plants: from mechanisms to ecological functions. Basic Appl Ecol 4:27-42

Dicke M, Van Loon JJA, Soler R (2009) Chemical complexity of volatiles from plants induced by multiple attack. Nat Chem Biol 5:317-324

Du Y, Poppy GM, Powell W, Pickett JA, Wadhams LJ, Woodcock CM (1998) Identification of semiochemicals released during aphid feeding that attract parasitoid Aphidius ervi. J Chem Ecol 24:1355-1368

Dudareva N, Negre F, Nagegowda DA, Orlova I (2006) Plant volatiles: recent advances and future perspectives. Crit Rev Plant Sci 25:417-440

Erb M, Foresti N, Turlings TC (2010) A tritrophic signal that attracts parasitoids to host-damaged plants withstands disruption by nonhost herbivores. BMC Plant Biol 10:247

Eriksson L, Byrne T, Johansson E, Trygg J, Vikström C (2013) Multiand megavariate data analysis: basic principles and applications, Ed 3rd revised edition. Umetrics Academy, Malmö, Sweden

Fäldt J, Arimura G, Gershenzon AJ, Takabayashi J, Bohlmann J (2003) Functional identification of AtTPSO3 as (E)-beta-ocimene synthase: a monoterpene synthase catalyzing jasmonate- and wound-induced volatile formation in Arabidopsis thaliana. Planta 216:745-751

Fisher AJ, Grimes HD, Fall R (2003) The biochemical origin of pentenol emission from wounded leaves. Phytochemistry 62:159-163

Gershenzon J, Croteau R (1991) Terpenoids. In: Rosenthal GA, Berenbaum MR (eds) Herbivores: their interactions with secondary plant metabolites. Acad. Press, New York, pp 165-219

Ginglinger JF, Boachon B, Höfer R, Paetz C, Köllner TG, Miesch L, Lugan R, Baltenweck R, Mutterer J, Ullmann P, Beran F, Claudel P, Verstappen F, Fischer MJ, Karst F, Bouwmeester H, Miesch M, Schneider B, Gershenzon J, Ehlting J, Werck-Reichhart D (2013) Gene coexpression analysis reveals complex metabolism of the monoterpene alcohol linalool in Arabidopsis flowers. Plant Cell 25:4640-4657

Girling RD, Stewart-Jones A, Dherbecourt J, Staley JT, Wright DJ, Poppy GM (2011) Parasitoids select plants more heavily infested with their caterpillar hosts: a new approach to aid interpretation of plant headspace volatiles. Proc R Soc B 278:2646-2653

Gols R, Roosjen M, Dijkman H, Dicke M (2003) Responses by jasmonic acid, low spider mite densities, or a combination of jasmonic acid treatment and spider mite infestation. J Chem Ecol 29:2651-2666

Heil M (2008) Indirect defence via tritrophic interactions. New Phytol 178:41-61
Hellemans J, Mortier G, De Paepe A, Speleman F, Vandesompele J (2007) qBase relative quantification framework and software for management and automated analysis of real-time quantitative PCR data. Genome Biol 8:R19

Herde M, Gartner K, Kollner TG, Fode B, Boland W, Gershenzon J, Gatz C, Tholl D (2008) Identification and regulation of TPS04/ GES, an Arabidopsis geranyllinalool synthase catalyzing the first step in the formation of the insect-induced volatile C16-homoterpene TMTT. Plant Cell 20:1152-1168

Hoedjes KM, Kruidhof HM, Huigens ME, Dicke M, Vet LEM, Smid HM (2011) Natural variation in learning rate and memory dynamics in parasitoid wasps: opportunities for converging ecology and neuroscience. Proc R Soc B Biol Sci 278:889-897

Houshyani B, Assareh M, Busquets A, Ferrer A, Bouwmeester HJ, Kappers IF (2013) Three-step pathway engineering results in more incidence rate and higher emission of nerolidol and improved attraction of Diadegma semiclausum. Metab Eng 15:88-97

Howe GA, Jander G (2008) Plant immunity to insect herbivores. Annu Rev Plant Biol 59:41-66

Huang M, Abel C, Sohrabi R, Petri J, Haupt I, Cosimano J, Gershenzon J, Tholl D (2010) Variation of herbivore-induced volatile terpenes among Arabidopsis ecotypes depends on allelic differences and subcellular targeting of two terpene synthases, TPS02 and TPS03. Plant Physiol 153:1293-1310

Kessler A, Baldwin IT (2002) Plant responses to insect herbivory: the emerging molecular analysis. Annu Rev Plant Biol 53:299-328

Kroes A, Van Loon JJA, Dicke M (2015) Density-dependent interference of aphids with caterpillar-induced defences in Arabidopsis: involvement of phytohormones and transcription factors. Plant Cell Physiol 56:98-106

Kusnierczyk A, Tran DHT, Winge P, Jorstad TS, Reese JC, Troczynska J, Bones AM (2011) Testing the importance of jasmonate signalling in induction of plant defences upon cabbage aphid (Brevicoryne brassicae) attack. BMC Genom 12:423

Liu P-P, Yang Y, Pichersky E, Klessig DF (2010) Altering expression of Benzoic Acid/Salicylic Acid Carboxyl Methyltransferase 1 compromises systemic acquired resistance and PAMP-triggered immunity in Arabidopsis. Mol Plant Microbe Int 23:82-90

Livak KJ, Schmittgen TD (2001) Analysis of relative gene expression data using real-time quantitative PCR and the $2^{-\Delta \Delta C T}$ method. Methods 25:402-408

Mewis I, Tokuhisa JG, Schultz JC, Appel HM, Ulrichs C, Gershenzon J (2006) Gene expression and glucosinolate accumulation in Arabidopsis thaliana in response to generalist and specialist herbivores of different feeding guilds and the role of defence signalling pathways. Phytochem 67:2450-2462

Moran PJ, Cheng Y, Cassell JL, Thompson GA (2002) Gene expression profiling of Arabidopsis thaliana in compatible plant-aphid interactions. Arch Insect Biochem 51:182-203

Mumm R, Dicke M (2010) Variation in natural plant products and the attraction of bodyguards involved in indirect plant defence. Can J Zool 88:628-667

Ohara Y, Takafuji A, Takabayashi J (2003) Response to host-infested plants in females of Diadegma semiclausum Hellen (Hymenoptera: Ichneumonidae). Appl Entomol Zool 38:157-162

Ozawa R, Arimura G, Takabayashi J, Shimoda T, Nishioka T (2000) Involvement of jasmonate- and salicylate-related signalling pathways for the production of specific herbivore-induced volatiles in plants. Plant Cell Physiol 41:391-398

Pangesti N, Weldegergis BT, Langendorf B, Van Loon JJA, Dicke M, Pineda A (2015) Rhizobacterial colonization of roots modulates plant volatile emission and enhances the attraction of a parasitoid wasp to host-infested plants. Oecologia 178:1169-1180

Pashalidou FG, Gols R, Berkhout BW, Weldegergis BT, Van Loon JJA, Dicke M, Fatouros NE (2015) To be in time: egg deposition 
enhances plant-mediated detection of young caterpillars by parasitoids. Oecologia 177:477-486

Pieterse CMJ, Van der Does D, Zamioudis C, Leon-Reyes A, Van Wees SCM (2012) Hormonal modulation of plant immunity. Annu Rev Cell Dev Biol 28:489-521

Ponzio C, Gols R, Pieterse CMJ, Dicke M (2013) Ecological and phytohormonal aspects of plant volatile emission in response to single and dual infestations with herbivores and phytopathogens. Funct Ecol 27:587-598

Ponzio C, Cascone P, Cusumano A, Weldegergis BT, Fatouros NE, Guerrieri E, Dicke M, Gols R (2016) Volatile-mediated foraging behaviour of three parasitoid species under conditions of dual insect herbivore attack. Anim Behav 111:197-206

Rodriguez-Saona C, Crafts-Brandner SJ, Canas LA (2003) Volatile emissions triggered by multiple herbivore damage: beet armyworm and whitefly feeding on cotton plants. J Chem Ecol 29:2539-2550

Rodriguez-Saona C, Chalmers JA, Raj S, Thaler JS (2005) Induced plant responses to multiple damagers: differential effects on an herbivore and its parasitoid. Oecologia 143:566-577

Schoonhoven LM, Van Loon JJA, Dicke M (2005) Insect-Plant Biology. Oxford University Press, Oxford

Snoeren TAL, Mumm R, Poelman EH, Yang Y, Pichersky E, Dicke M (2010) The herbivore-induced plant volatile methyl salicylate negatively affects attraction of the parasitoid Diadegma semiclausum. J Chem Ecol 36:479-489

Soler R, Badenes-Perez FR, Broekgaarden C, Zheng SJ, David A, Boland W, Dicke M (2012) Plant-mediated facilitation between a leaf-feeding and a phloem-feeding insect in a brassicaceous plant: from insect performance to gene transcription. Funct Ecol 26:156-166

Stam JM, Kroes A, Li Y, Gols R, Van Loon JJA, Poelman EH, Dicke M (2014) Plant interactions with multiple insect herbivores: from community to genes. Annu Rev Plant Biol 65:689-713

Tholl D, Lee S (2011) Terpene specialized metabolism in Arabidopsis thaliana. The Arabidopsis Book 9:e0143. doi:10.1199/tab.0143

Tholl D, Chen F, Petri J, Gershenzon J, Pichersky E (2005) Two sesquiterpene synthases are responsible for the complex mixture of sesquiterpenes emitted from Arabidopsis flowers. Plant J 42:757-771
Truong D-H, Heuskin S, Delaplace P, Francis F, Lognay G (2014) VOC emissions and protein expression mediated by the interactions between herbivorous insects and Arabidopsis plant. A review. Biotechnol Agron Soc Environ 18:455-464

Turlings TCJ, Wäckers FL, Vet LEM, Lewis WJ, Tumlinson JH (1993) Learning of host-finding cues by hymenopterous parasitoids. In: Papaj DR, Lewis AC (eds) Insect learning: ecological and evolutionary perspectives. Chapman \& Hall, New York, pp 51-78

Turlings TCJ, Bernasconi M, Bertossa R, Bigler F, Caloz G, Dorn S (1998) The induction of volatile emissions in maize by three herbivore species with different feeding habits: possible consequences for their natural enemies. Biol Control 11:122-129

Van Poecke RMP (2007) Arabidopsis-insect interactions. In: Somerville CR, Meyerowitz EM (eds) The Arabidopsis book. American Society of Plant Biologists, Rockville. doi:10.1199/tab.0107

Van Poecke RMP, Posthumus MA, Dicke M (2001) Herbivore-induced volatile production by Arabidopsis thaliana leads to attraction of the parasitoid Cotesia rubecula: chemical, behavioral, and geneexpression analysis. J Chem Ecol 27:1911-1928

Van Schie CC, Haring MA, Schuurink RC (2007) Tomato linalool synthase is induced in trichomes by jasmonic acid. Plant Mol Biol 64:251-263

Vandesompele J, De Preter K, Pattyn F, Poppe B, Van Roy N, De Paepe A, Speleman F (2002) Accurate normalization of real-time quantitative RT-PCR data by geometric averaging of multiple internal control genes. Genome Biol 3:1-12

Wei J, Van Loon JJA, Gols R, Menzel TR, Li N, Kang L, Dicke M (2014) Reciprocal crosstalk between jasmonate and salicylate defence-signalling pathways modulates plant volatile emission and herbivore host-selection behaviour. J Exp Bot 65:3289-3298

Zhang PJ, Zheng SJ, Van Loon JJA, Boland W, David A, Mumm R, Dicke M (2009) Whiteflies interfere with indirect plant defence against spider mites in Lima bean. Proc Natl Acad Sci USA 106:21202-21207

Zhang PJ, Broekgaarden C, Zheng SJ, Snoeren TAL, Van Loon JJA, Gols R, Dicke M (2013) Jasmonate and ethylene signalling mediate whitefly-induced interference with indirect plant defence in Arabidopsis thaliana. New Phytol 197:1291-1299 